\title{
COHOMOLOGY, MAXIMAL IDEALS, AND POINT EVALUATIONS ${ }^{1}$
}

\author{
ALEXANDER NAGEL ${ }^{2}$
}

\begin{abstract}
We consider algebras $A$ of continuous complex valued functions, which are given as the set of global sections of a sheaf $\mathscr{S}$ on a topological space $X$. Under the hypothesis that all the higher cohomology groups of the sheaf are zero, we investigate the relationship between ideals in $A$, kernels of algebra homomorphisms of $A$ into the complex numbers $C$, and sets of functions vanishing at a point of $X$. As applications, we obtain some simple proofs of theorems about ideals in certain algebras of holomorphic functions.
\end{abstract}

Let $X$ be a topological space, and let $\mathscr{S}$ be a sheaf of local $C$-algebras on $X$. We shall assume:

(a) For all $x \in X$, the maximal ideal of the stalk $\mathscr{S}_{x}$ is $m_{x}$, and the

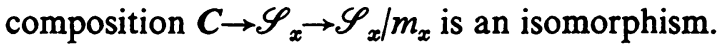

(b) For every $f \in \Gamma(X, \mathscr{S})$, the associated complex valued function $\hat{f}$ is continuous, where $\hat{f}(x)$ is the residue class of the germ of $f$ at $x$ in $\mathscr{S}_{x} / m_{x}$.

(c) For all $q \geqq 1, H^{q}(X, \mathscr{S})=(0)$.

THEOREM 1. Suppose that $I$ is an ideal in $\Gamma(X, \mathscr{S})$, and that there is a finite subset $\left\{f_{1}, \cdots, f_{n}\right\} \subset I$ so that for all $x \in X$, there exists a $j$ so that $f_{j}(x) \neq 0$. Then $I=\Gamma(X, \mathscr{S})$.

ProOf. Consider the following Koszul complex of sheaves on $X$ :

$$
\begin{aligned}
(0) & \rightarrow \bigwedge^{n} \mathscr{S}^{n} \rightarrow^{\delta_{n}} \bigwedge^{n-1} \mathscr{S}^{n} \rightarrow \cdots \\
& \rightarrow \bigwedge^{k} \mathscr{S}^{n} \rightarrow^{\delta_{k}} \bigwedge^{k-1} \mathscr{S}^{n} \rightarrow \cdots \\
& \rightarrow \bigwedge^{2} \mathscr{S}^{n} \rightarrow^{\delta_{2}} \mathscr{S}^{n} \rightarrow^{\delta_{1}} \mathscr{S} \rightarrow(0)
\end{aligned}
$$

Here $\mathscr{S}^{n}$ denotes the direct sum of $n$ copies of $\mathscr{S}$, and $\wedge^{k} \mathscr{S}^{n}$ denotes the $k$ th exterior power of $\mathscr{S}^{n}$. Also:

$$
\delta_{1}\left(s_{1}, \cdots, s_{n}\right)=\sum_{i=1}^{n} s_{i} f_{i},
$$

Received by the editors January 29, 1973 and, in revised form, March 26, 1973.

AMS (MOS) subject classifications (1970). Primary 14F05, 46J20.

Key words ard phrases. Cohomology, maximal ideals, point evaluation, Koszul complex, analytic functions, domain of holomorphy, Stein space.

${ }^{2}$ This paper incorporates part of the author's doctoral dissertation at Columbia University.

${ }^{2}$ This work was supported in part by NSF Grant GP-33029.

(c) American Mathematical Society 1974 
and

$$
\begin{aligned}
\delta_{k}\left(s_{i_{1}, \cdots, i_{k}}\left[i_{1}\right] \wedge\right. & \left.\cdots \wedge\left[i_{k}\right]\right) \\
& =(-1)^{j-1} s_{i_{1}, \cdots, i_{k}} f_{i_{j}}\left[i_{1}\right] \wedge \cdots \wedge\left[i_{j}\right] \wedge \cdots \wedge\left[i_{k}\right] .
\end{aligned}
$$

Here $\left\{\left[i_{1}\right] \wedge \cdots \wedge\left[i_{k}\right]\right\}$ are the basis elements of $\wedge^{k} \mathscr{S}^{n}$ over $\mathscr{S} .\left[i_{j}\right]^{\wedge}$ indicates the omission of $\left[i_{j}\right]$. Standard repeated index summation notation has been used.

We now show that sequence (1) is exact. First, an easy computation shows that $\delta_{k-1} \delta_{k}=0$, so that sequence (1) is actually a complex. Next, at each point $x \in X$ at least one of the functions $\hat{f}_{1}, \cdots, \hat{f}_{n}$ is not zero. Suppose $\hat{f}_{l}(x) \neq 0$. Then $\left[f_{l}\right]_{x} \notin m_{x}$, so $\left[f_{l}\right]_{x}^{-1} \in \mathscr{S}_{x}$ exists. Hence given $s \in \mathscr{S}_{x}, s=f_{l} \cdot\left(s \cdot\left[f_{l}\right]_{x}^{-1}\right)$. Thus $\delta_{1}$ is surjective.

More generally, let $\omega=s_{i_{1}, \ldots, i_{k}}\left[i_{l}\right] \wedge \cdots \wedge\left[i_{k}\right] \in\left(\bigwedge^{k} \mathscr{S}^{n}\right)_{x}$ and suppose $\delta_{k} \omega=0$. Let $\varphi \in\left(\bigwedge^{k+1} \mathscr{S}^{n}\right)_{x}$ be the element

$$
\varphi=(-1)^{k}\left[f_{l}\right]_{x}^{-1} s_{i_{1}, \cdots, i_{k}}\left[i_{1}\right] \wedge \cdots \wedge\left[i_{k}\right] \wedge[l] .
$$

Then another computation shows that $\delta_{j+1}(\varphi)=\omega$. Thus sequence (1) is exact.

Each term in (1) is isomorphic to a direct sum of a finite number of copies of $\mathscr{S}$. Since $H^{q}(X, \mathscr{S})=(0)$ for all $q \geqq 1$, the higher cohomology of each term in (1) vanishes. Hence in sequence (1), if we take global sections, the resulting sequence is exact. In particular, we have

$$
\Gamma(X, \mathscr{S})^{n} \rightarrow^{\delta_{1}} \Gamma(X, \mathscr{S}) \rightarrow(0)
$$

exact; i.e. the map $\delta_{1}: \Gamma(X, \mathscr{S})^{n} \rightarrow \Gamma(X, \mathscr{S})$ given by $\delta_{1}\left(s_{1}, \cdots, s_{n}\right)=$ $\sum_{j=1}^{n} s_{j} f_{j}$ is surjective. Since $\left\{f_{1}, \cdots, f_{n}\right\} \subset I$ it follows that $I=\Gamma(X, \mathscr{S})$, and hence the theorem is true.

Corollary 1. Suppose that $X$ is compact. Then for every proper ideal $I \subsetneq \Gamma(X, \mathscr{S})$, there exists an $x \in X$ so that $\hat{f}(x)=0$ for all $f \in I$.

Proof. Suppose the theorem is false: Then for every $x \in X$, there exists $f \in I$ with $\hat{f}(x) \neq 0$. Since $\hat{f}$ is continuous, there is an open neighborhood $N$ of $x$ in $X$ so that $\hat{f}(y) \neq 0$ for all $y \in N$. By compactness, we can find a finite number of these neighborhoods which cover $X$. Hence there is a finite set $\left\{f_{1}, \cdots, f_{n}\right\} \subset I$ so that for any $y \in X$, there is an $f_{j}$ with $\hat{f}_{j}(y) \neq 0$. By Theorem 1, this implies that $I=\Gamma(X, \mathscr{S})$, so that the corollary is true.

COROLlaRY 2. Let $X \subset C^{n}$ be a compact set which can be written $X=\bigcap_{i=1}^{\infty} D_{i}$, where $D_{i} \subset C^{n}$ is a domain of holomorphy. Let $\mathscr{H}(X)$ be the algebra of germs of holomorphic functions on $X$. Then every maximal ideal of $\mathscr{H}(X)$ is the set of functions vanishing at some fixed point of $X$. 
ProOf. Let $\mathcal{O}$ denote the sheaf of germs of holomorphic functions on $C^{n}$. Then $\mathscr{H}(X)=\Gamma(X, \mathcal{O})$, and for $q \geqq 1, H^{q}(X, \mathcal{O})=\operatorname{dir} \lim H^{q}\left(D_{i}, \mathcal{O}\right)$ $=(0)$ by Cartan's Theorem B. (See Gunning and Rossi [1, p. 243].) Since $X$ is compact, the corollary follows from Corollary 1 .

COROLlaRY 3. Let $X \subset C^{n}$ satisfy the same conditions as in Corollary 2. Let $A(X)$ denote the uniform closure of $\mathscr{H}(X)$. Then the maximal ideal space of $A(X)$, viewed as a function algebra, is homeomorphic to $X$.

Proof. It suffices to show that for every algebra homomorphism $\varphi$ : $A(X) \rightarrow C$, there exists $x \in X$ so that $\varphi(f)=f(x)$. Let $I=\{f \in \mathscr{H}(X) \mid \varphi(f)=0\}$. Then $I$ is a maximal ideal in $\mathscr{H}(X)$ so by Corollary 1 , there is $x \in X$ with $\varphi(f)=f(x)$ for all $f \in \mathscr{H}(X)$. Since $\mathscr{H}(X)$ is dense in $A(X)$, and $\varphi$ is continuous, it follows that $\varphi(f)=f(x)$ for all $f \in A(X)$.

Corollaries 2 and 3 are false without the hypothesis that $X=\bigcap_{i=1}^{\infty} D_{i}$, where each $D_{i}$ is a domain of holomorphy, as the standard example $X=\left\{(z, w) \in C^{2}|| z|\leqq| w \mid \leqq 1\right\}$ shows. Also, Corollary 1 is false in general if $X$ is not compact. For example let $X=C$, and $\mathscr{S}=\mathcal{O}$, the sheaf of germs of holomorphic functions on $C$. Then $H^{q}(X, \mathscr{S})=(0)$ for $q \geqq 1$ and $\Gamma(X, \mathscr{S})$ is the algebra of entire functions. However if $\left\{z_{n}\right\}$ is an infinite discrete set in $C$, and $I$ is the ideal of entire functions which vanish at all except some finite subset of $\left\{z_{n}\right\}$, then $I$ is a proper ideal, but the functions in $I$ have no common zero. We can, however, prove the following in the noncompact case:

Corollary 4. Let $X$ and $\mathscr{S}$ satisfy conditions (a), (b), (c). Suppose there are a finite number of global sections $\left\{f_{1}, \cdots, f_{n}\right\} \subset \Gamma(X, \mathscr{S})$ such that the associated continuous functions on $X$ separate the points of $X$. Then for every nonzero algebra homomorphism $\varphi: \Gamma(X, \mathscr{S}) \rightarrow C$, there exists $x \in X$ so that $\varphi(f)=\hat{f}(x)$ for all $f \in \Gamma(X, \mathscr{S})$.

Proof. Let $g_{j}=f_{j}-\varphi\left(f_{j}\right)$, for $j=1, \cdots, n$. Then the functions $\hat{g}_{j}$, $j=1, \cdots, n$, still separate the points of $X$. Let $Z=\left\{x \in X \mid \hat{g}_{j}(x)=0\right\}$. Then there is at most one point $x_{0} \in Z$. If the corollary is not true, there exists $g_{0} \in \Gamma(X, \mathscr{S})$ so that $\varphi\left(g_{0}\right)=0$ but $\hat{g}_{0}\left(x_{0}\right) \neq 0$. Then the functions $\left\{\hat{g}_{0}, \cdots, \hat{g}_{n}\right\}$ have no common zeros. Proceeding as in Theorem 1 , we see that every element $f \in \Gamma(X, \mathscr{S})$ can be written $\sum_{i=0}^{n} f_{i} g_{i}$ for some $f_{i} \in \Gamma(X, \mathscr{S})$. But then $\varphi(f)=\sum_{i=0}^{n} \varphi\left(f_{i}\right) \varphi\left(g_{i}\right)=0$, so $\varphi$ is the zero homomorphism, a contradiction. Hence the corollary is true.

The following result is now immediate:

COROLlary 5. Let $X$ be a Stein space supporting a finite number of holomorphic functions separating points, and let $\mathcal{O}$ be the structure sheaf. Then every nonzero algebra homomorphism $\varphi: \Gamma(X, \mathcal{O}) \rightarrow C$ is given by 
evaluation at a point of $X$. In particular, every algebra homomorphism is continuous if $\Gamma(X, \mathcal{O})$ is given the usual Fréchet topology.

Finally, we show that, at least without additional assumptions on the sheaf $\mathscr{S}$, the converse to Corollary 1 is not in general true. Let $X=\{z \in C|1 \leqq| z \mid \leqq 2\}$. Let $\mathscr{S}$ be the sheaf of nonsingular rational functions on $X$. Then $\Gamma(X, \mathscr{S})$ is the algebra of rational functions on $C$ having no poles on $X$. It is easy to see that for any proper ideal $I \subset \Gamma(X, \mathscr{S})$, there exists $\lambda \in X$, so that all the functions in $I$ vanish at $\lambda$.

Now let $. U_{1}=\left\{z \in X \mid \operatorname{Re}(z)<\frac{1}{2}\right\}$, and $U_{2}=\left\{z \in X \mid \operatorname{Re}(z)>-\frac{1}{2}\right\}$. Then $\left\{U_{1}, U_{2}\right\}$ is an open cover of $X$, and $U_{1} \cap U_{2}$ is the disjoint union of two open sets in $X$. Define $f_{12}$ in $U_{1} \cap U_{2}$ by setting

$$
\begin{aligned}
f_{12}(z) & =0, & & \text { if } \operatorname{Im}(z)<0, \\
& =z, & & \text { if } \operatorname{Im}(z)>0 .
\end{aligned}
$$

Set $f_{21}=-f_{12}$. Then $\left\{f_{i j}\right\}$ is a 1-cocycle with values in $\mathscr{S}$. If $\left\{f_{i j}\right\}$ were a coboundary on some refinement, $f_{i j}=F_{i}-F_{j}$, then $F_{i}=F_{j}$ when $\operatorname{Im}(z)<0$, so we would have a multiple valued rational function, which is impossible. Hence $H^{1}(X, \mathscr{S}) \neq(0)$.

\section{REFERENCE}

1. R. C. Gunning and H. Rossi, Analytic functions of several complex variables, Prentice-Hall Series in Modern Analysis, Prentice-Hall, Englewood Cliffs, N.J., 1965. MR 31 \#4927.

Department of Mathematics, University of Wisconsin, Madison, Wisconsin 53706 